\title{
Modeling of Porous Insertion Electrodes with Liquid Electrolyte
}

\author{
West, Keld; Jacobsen, Torben; Atlung, Sven
}

Published in:

Journal of The Electrochemical Society

Link to article, DOI:

$10.1149 / 1.2124188$

Publication date:

1982

Document Version

Publisher's PDF, also known as Version of record

Link back to DTU Orbit

Citation (APA):

West, K., Jacobsen, T., \& Atlung, S. (1982). Modeling of Porous Insertion Electrodes with Liquid Electrolyte. Journal of The Electrochemical Society, 129(7), 1480-1485. https://doi.org/10.1149/1.2124188

\section{General rights}

Copyright and moral rights for the publications made accessible in the public portal are retained by the authors and/or other copyright owners and it is a condition of accessing publications that users recognise and abide by the legal requirements associated with these rights.

- Users may download and print one copy of any publication from the public portal for the purpose of private study or research.

- You may not further distribute the material or use it for any profit-making activity or commercial gain

- You may freely distribute the URL identifying the publication in the public portal

If you believe that this document breaches copyright please contact us providing details, and we will remove access to the work immediately and investigate your claim 
$\eta_{t}(\exp ),\left[\eta_{t}(\right.$ calc $\left.)\right]$ experimental (calculated) value of $\eta$ at time $t$

$\lambda \quad \equiv\left|\left(\Delta i_{0} / i_{0}\right) /\left(\Delta C_{\mathrm{d} 1} / C_{\mathrm{d} 1}\right)\right|$

$\lambda\left(T_{1}\right),\left[\lambda\left(T_{c}\right)\right]$ value of $\lambda$ at the optimal time scale for measuring $i_{0}\left[C_{\mathrm{dl}}\right]$

$\nu \quad$ stoichiometric number

$\nu_{0},\left[\nu_{R}\right]$ stoichiometric coefficients of $O[R]$ in the overall electrode reaction

o correlation coefficient between $i_{0}$ and $C_{\mathrm{dl}}$

${ }_{2} \sigma(p)^{2},\left[1 \sigma(p)^{2}\right]$ population variance of parameter $p$ in a two-estimate (single-estimate) system. The presubscript stands for the number of estimates. In case no subscript is specified, the number of estimates is either one or two depending on the meanings of the other parameters in the equation population variance of $\eta$

$\sigma(\eta)^{2}$
$\tau_{c},\left[\tau_{d}\right]$ charge-transfer [diffusional] relaxation constant

\section{REFERENCES}

1. T. Berzins and P. Delahay, J. Am. Chem. Soc., 77, $6448(195 \bar{\jmath})$.

2. E. Yeager and J. Kuta, in "Physical Chemistry,
An Advanced Treatise," Vol. IX A, H. Eyring, D. Henderson, and W. Fost, Editors, Academic Press, New York (1970)

3. D. J. Kooijman and J. H. Sluyters, Electrochim. Acta, 12, 693 (1967)

4. D. J. Kooijman and J. H. Sluyters, ibid., 12, 1579 (1967).

5. D. J. Kooijman, J. Electroanal. Chem. Interfacial Electrochem., 18, 81 (1968).

6. H. P. Van Leeuwen, D. J. Kooijman, M. SluytersRehbach, and J. H. Sluyters, ibid., 23, 475 (1969)

7. H. Reller and E. Kirowa-Eisner, ibid., 103, 335 (1979).

8. H. Reller and E. Kirowa-Eisner, This Journal, 127, 1725 (1980).

9. W. D. Cooper and R. Parsons, Trans. Faraday Soc., 66, 1698 (1970).

10. F. James and M. Roos, Comp. Phys. Commun., 10, $343(1975)$.

11. W. T. Eadie, D. Drijard, F. E. James, M. Roos, and B. Sadoulet, "Statistical Methods in Experimental Physics," North-Holland Pub. Co., Amsterdam (1971).

\title{
Modeling of Porous Insertion Electrodes with Liquid Electrolyte
}

\author{
K. West, T. Jacobsen, ${ }^{*}$ and S. Atlung ${ }^{*}$ \\ Fysisk-Kemisk Institut, The Technical University of Denmark, DK-2800 Lyngby, Denmark
}

\section{ABSTRACT}

The dynamics of porous insertion electrodes during charge or discharge is described by a simplified mathematical model, accounting for the coupled transport in electrode and electrolyte phases. A numerical method to evaluate the response of this model to either controlled potential or controlled current is outlined, and numerical results for the discharge of a porous $\mathrm{TiS}_{2}$-electrode in an idealized organic electrolyte are presented. It is demonstrated how electrolyte depletion is the principal limiting factor in the capacity obtained during discharge of this electrode system. This depletion is a consequence of the mobility of the ions not inserted, hence the performance of this type of electrode is optimized by choosing electrolytes with transport number as close to unity as possible for the inserted ion.

One of the consequences of the energy crisis is a renewed interest in secondary batteries for traction purposes. As a partial electrification of the transport sector would make the energy consumption pattern of the community more flexible, and thus less sensitive to changes in the availability of the different fuels, much effort is invested in development of battery systems for this purpose.

Parallel to the continuous development of existing battery systems, much research is devoted to alkali metal batteries because of the high energy density offered by these systems. One of the systems considered to have potential application as a high rate battery is a negative lithium or lithium alloy electrode couple with a positive insertion electrode $1(1,2)$. The insertion materials under investigation for this purpose are primarily layered transition metal dichalcogenides [e.g., $\mathrm{TiS}_{2}$ (3), $\mathrm{VSe}_{2}$ (4)] and oxide framework structures like $\mathrm{V}_{2} \mathrm{O}_{5}$ (5), $\mathrm{V}_{6} \mathrm{O}_{13}$ (6), and recently $(\mathrm{Mo}, \mathrm{V})_{2} \mathrm{O}_{5}(7)$, but also amorphous chalcogenides (8) have received some interest.

Until now no entirely satisfactory combination of electrodes and electrolytes has been found, although it has been demonstrated ( 9 ) that a lithium/organic electrolyte/titanium disulfide battery could be adapted to electric vehicle propulsion. In any case, the development of new electrode and electrolyte materials is proceeding very fast, and it is of importance to obtain a better understanding of the transport processes in this

- Electrochemical Society Active Member.

Key words: intercalation, battery capacity, simulation.

1 The term "insertion electrode" used here covers as well intercalation of a "guest" species in a "host" lattice, as the formation of solid solutions during the electrode process. type of batteries in order to anticipate the combinations of electrode and electrolyte properties that can meet the requirements corresponding to a given set of battery specifications.

In a previous paper (10) thin, nonporous insertion electrodes were treated in detail. In the present paper a mathematical description of porous insertion electrodes is presented to extend these calculations to more realistic electrodes.

Porous electrode theory has been reviewed by deLevie (11) and more recently by Newman and Tiedemann (12). The porous insertion electrode is, however, different from the electrode types treated previously, as it involves transport in both electrolyte and electrode phases. These two transport systems are coupled both through mass balances and through the variation of the electrode potential with the surface composition of the electrode phase. This variation is typically large for intercalation compounds (hundreds of millivolts) and current distribution in the pores due to charge transfer resistance is of minor importance compared to the effect of the varying surface composition. The transient behavior of porous electrodes with low charge transfer resistance has been treated by Pollard and Newman (14) for the case where the equilibrium electrode potential is not a function of the utilization of the electrode material.

\section{Model Formulation}

An electrode with irregularly shaped pores and cavities will be difficult if not impossible to describe mathematically. Therefore, we have chosen to model the porous insertion electrode with systems of much 
simpler geometry, but still possessing the important functional features of the real electrodes. The geometry of these models is shown in Fig. 1 and 2.

Each of these model systems consist of a porous slab of electrode material of high electronic conductivity and of thickness $l$. On one side $(z=0)$ this slab is contacted by a metallic conductor serving as current collector, and on the other $(z=l)$ by an electrolyte also filling the pores of the electrode. This electrolyte is considered to be liquid with mobile anions and cations.

The three model geometries consist of equally sized particles uniformly distributed in the electrode and of one of the following shapes: parallel sheets (Fig. 1); long cylinders (transport across the ends of the cylinders should be negligible) (Fig. 2a); and spheres (Fig. 2b).

The packing of the cylinders or the spheres should ensure that all particles are in electronic contact with the current collector, and they should be evenly distributed so the porosity does not change significantly in any directions within the electrode. There are no restrictions on the orientation of the cylinders, but as the concentration will be taken to be constant around a cross section of the particles, the diameter of the cylinders or the spheres should be small compared to the thickness of the electrode.

In order to keep the transport equations from getting too involved and to keep the computational work at a reasonable level, the model systems are simplified further by the following assumptions:

1. The particle-particle distance (the width of the pores) is so small compared with the thickness of the electrode that the potential and concentrations in the pores can be considered as varying in one dimension only (i.e., along the length of the pore).

2. The ionic conductance of the electrode phase is low compared with the conductance of the electrolyte, so diffusion in the electrode phase parallel to the surface of the electrode particle will not contribute significantly to the overall transport. Solid-state diffu-

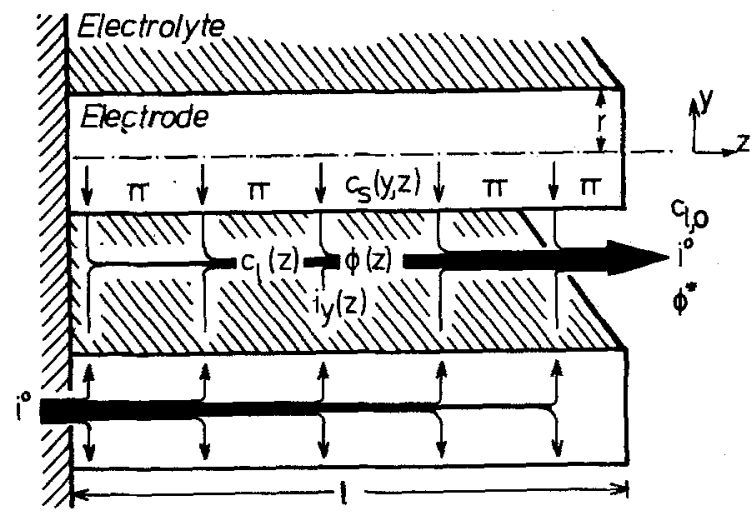

Fig. 1. Schematic diogram of electrode model (plane geometry). Arrows indicate directions of fluxes considered.

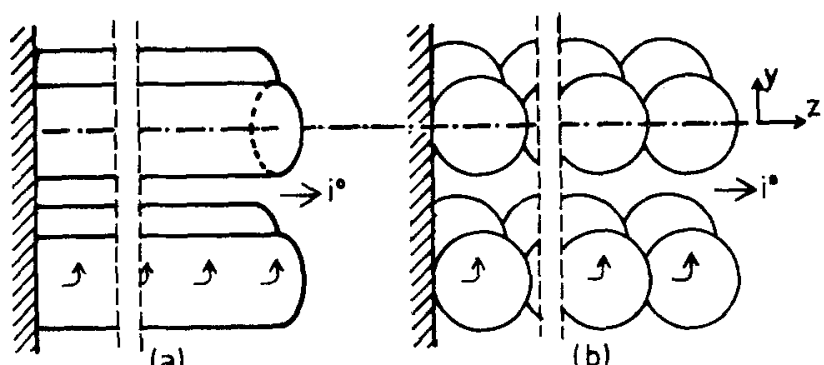

Fig. 2. Schematic diagrams of electrode models: (a) cylindrical geometry; (b) spherical geometry. sion in the electrode phase thus only removes the inserted species from the electrode/electrolyte interfaces as indicated in Fig. 1.

3. Space charge accumulation is negligible. This implies that the electroneutrality principle can be applied in the derivation of the transport equations instead of the more involved Poisson-Boltzmann equation.

4. Charge transfer overvoltages are negligible.

5. The electronic conductivity of the electrode phase is so high that the Fermi potential $(\pi)$ in this phase is constant.

6. The electrolyte salt considered is mono-mono valent.

7. Volume flow due to expansion of the solid matrix and concentration changes in the electrolyte are neglected.

\section{Basic Equations}

On the basis of these assumptions the equations governing transport in the model pore system can be formulated. As there is no significant transport parallel to the surface of the electrode particles, the diffusion equation for the solid phase will be one-dimensional in the coordinate system belonging to the symmetry class of the electrode particles (cartesian, cylindrical, or spherical, respectively). The electric field in this phase is small, and the diffusion can be treated according to the Fick laws. With concentration and chemical diffusion coefficient of the inserted species designated by $c_{\mathrm{s}}$ and $D_{\mathrm{s}}$ and the time by $t$, the diffusion equation is

$$
\frac{\partial c_{\mathrm{s}}(y, z)}{\partial t}=\nabla \cdot D_{\mathrm{s}} \nabla_{\mathrm{y}} c_{\mathrm{s}}(y, z)
$$

where $\nabla_{\mathrm{y}}$ is the nabla-operator for the direction perpendicular to the particle surface. The boundary conditions in the center $(y=0)$ and at the surface of the electrode particles $(y=r)$ are

$$
\begin{gathered}
\left.\nabla_{\mathrm{y}} \mathrm{c}_{\mathrm{s}}(y, z)\right|_{y=0}=0 \\
\left.\nabla_{\mathrm{y}} \mathrm{c}_{\mathrm{s}}(y, z)\right|_{y=r}=-i_{\mathrm{y}}(z) / \mathbf{F} D_{\mathrm{s}}
\end{gathered}
$$

where $i_{y}$ is the current density across the particle/ electrolyte interface.

The transport in the electrolyte will be treated as one-dimensional diffusion and migration according to the Nernst-Planck equation. For the cations an additional term must be included in this equation to account for the flux of cations across the particle/electrolyte interface (given by the transfer current density $\left.i_{y}\right)$. Due to electroneutrality the anion and cation concentrations are equal $\left(c_{1}\right)$

$$
\begin{aligned}
& \frac{\partial c_{1}(z)}{\partial t}=\frac{\partial}{\partial z} D_{-}\left[\frac{\partial c_{1}(z)}{\partial z}-c_{l}(z) \cdot \frac{\mathbf{F}}{R T} \frac{\partial \phi(z)}{\partial z}\right] \\
& \frac{\partial c_{1}(z)}{\partial t}=\frac{\partial}{\partial z} D_{+}\left[\frac{\partial c_{1}(z)}{\partial z}\right. \\
& \left.+c_{1}(z) \cdot \frac{\mathbf{F}}{R T} \frac{\partial \phi(z)}{\partial z}\right]+i_{\mathrm{y}}(z) g / \mathbf{F}
\end{aligned}
$$

where $D_{+}$and $D_{-}$are diffusion coefficients of cations and anions, respectively, and $\phi$ is the electric potential in the electrolyte. The geometric factor $g$ is the average ratio between the circumference and the crosssectional area of the pore.

The boundary conditions for these equations at the bottom of the pore $(z=0)$ and at the electrode surface $(z=l)$ are

$$
\begin{gathered}
\left.\frac{\partial c_{1}}{\partial z}\right|_{z=0}=\left.\frac{\partial \phi}{\partial z}\right|_{z=0}=0 \\
c_{1}(l)=c_{1,0} ; \phi(l)=\phi^{*}
\end{gathered}
$$


where $c_{1,0}$ and $\phi^{*}$ are constants or specified functions of time. Furthermore it is assumed that the electrode initially is in equilibrium

$$
\begin{aligned}
t=0 \Rightarrow c_{1} & =c_{1,0} \text { and } \phi=\phi^{\circ}(\text { all } z) \\
c_{\mathrm{s}} & =c_{\mathrm{s}, 0} \quad(\text { all } y \text { and } z)
\end{aligned}
$$

where $\phi^{0}$ is given by the equilibrium electrode potential $\left(\pi-\phi^{0}\right)$ corresponding to the composition $c_{1,0}$, $c_{s, 0}$.

The last constraint necessary to couple the electrode and electrolyte systems is the potential relation

$$
(\pi-\phi(z))=h\left(c_{\mathrm{s}}^{*}(z), c_{1}(z)\right)
$$

where $c_{s}{ }^{*}(z)$ is the local surface concentration of the inserted ion. As local electrochemical equilibrium at the electrode/electrolyte interface is assumed (no charge transfer overvoltage), $h$ is a single valued function of $c_{s}{ }^{*}$ and $c_{1}$ only. The exact form of this function $h$ will depend on the electrode material chosen. In many instances, however, a function of the following type will give a reasonable good approximation (13)

$$
\begin{aligned}
(\pi-\phi)=(\pi-\phi)^{\theta} & +\frac{R T}{F}\left(\ln \left(\left(c_{\mathrm{s}}{ }^{0}-c_{\mathrm{s}}{ }^{*}\right) / c_{\mathrm{s}}{ }^{*}\right)\right. \\
& \left.+\ln \left(c_{1}\right)-f\left(c_{\mathrm{s}}{ }^{*} / c_{\mathrm{s}}{ }^{0}\right)-0.5\right)
\end{aligned}
$$

where $c_{s}{ }^{0}$ is the saturation concentration of the inserted species in the electrode phase. $(\pi-\phi)^{\theta}$ and $f$ are characteristic constants of the electrode material. Applying this expression implies that the insertion process is equivalent to an adsorption process with linear interaction term (Frumkin isotherm).

As $\pi$ is constant in space, $\partial \phi(z) / \partial z$ in Eq. [4] and [5] can be replaced by $-\partial(\pi-\phi(z)) / \partial z$, and the space and time variations of concentrations and potential in the model pore system are thus determined by [1], [4], [5], and [9] together with the appropriate boundary conditions.

\section{Dimensionless Parameters}

In the limited universe of a numerical model it is often inconvenient to measure quantities relative to standards (e.g., SI-units) which are not part of the model. Often it is an advantage to measure the quantities relative to some of the characteristic parameters of the system and transform the equations into dimensionless forms. With a proper choice of reference parameters, the dimensionless variables can give a simpler description of the state of the system than the corresponding dimensioned variables.

A dimensionless form of Fick's laws where time is measured relative to a time constant of diffusion and length relative to the dimension of the diffusion region is often used (15). Considering diffusion in the electrode phase, this transformation gives the dimensionless time and length

$$
T=t D_{s} \% / r^{2} ; \quad Y=y / r
$$

where $D_{\mathrm{s}}{ }^{\circ}$ is a characteristic value of the diffusion coefficient of the inserted ion.

Measuring the concentration of the inserted ion relative to its saturation concentration $X=c_{\mathrm{s}} / \mathrm{c}_{\mathrm{s}}{ }^{\circ}$, the transport equation for the electrode phase [1], assumes the following form

$$
\frac{\partial}{\partial T} X(Y)=\nabla \cdot U_{s} \nabla_{Y} X(Y)
$$

where $U_{s}$ is a dimensionless mobility

$$
U_{\mathrm{s}}=D_{\mathrm{s}} / D_{\mathrm{s}}{ }^{\circ}
$$

The dimensionless electrode current density or cation flux density across the electrode/electrolyte in- terface corresponding to this transformation is defined as

$$
I_{\mathrm{y}}=i_{\mathrm{y}} \cdot r \cdot n_{\mathrm{g}} / \mathbf{F} \cdot c_{\mathrm{s}}{ }^{\mathrm{o}} \cdot D_{\mathrm{s}}^{\mathrm{o}}
$$

Noting that the surface to volume ratio of the three types of electrode particles considered is $n_{\mathrm{g}} / r$, where $n_{\mathrm{g}}$ is 1,2 , or 3 for planar, cylindrical, or spherical geometry, respectively, it can be seen that $I_{y}$ is the transfer current density measured relative to the specific charge density available in the electrode phase and to the time constant of diffusion.

The transport equations for the electrolyte [4] and [5] must be transformed in a slightly different way, utilizing the dimensionless time $T$ already defined in Eq. [11]. Introducing the dimensionless variables

$$
C=c_{\mathfrak{l}} / c_{\mathfrak{l}, 0} ; \quad E=(\pi-\phi) \mathbf{F} / R T ; \quad Z=z / l
$$

and the dimensionless mobilities

$$
U_{+}=\left(D_{+} / D_{\mathrm{s}}{ }^{0}\right)(l / r)^{2} ; \quad U_{-}=\left(D_{-} / D_{\mathrm{s}}{ }^{\circ}\right)(l / r)^{2}
$$

the equations governing transport in the electrolyte assume the following nondimensional forms

$$
\frac{\partial C}{\partial T}=\frac{\partial}{\partial Z} U_{-}\left(\frac{\partial C}{\partial Z}+C \frac{\partial E}{\partial Z}\right)
$$

and

$$
\frac{\partial C}{\partial T}=\frac{\partial}{\partial Z} U+\left(\frac{\partial C}{\partial Z}-C \frac{\partial E}{\partial Z}\right)+I_{y} G
$$

The dimensionless geometric factor $G=g \cdot r \cdot c_{s} \%$ $\left(c_{1,0} \cdot n_{g}\right)$ contains information on the "shape" of the pores. For the simple geometries described above, $\boldsymbol{g}$ is equal to $n_{\mathrm{g}}(1-P) /(r \cdot P)$, and $G$ 's dependence on system parameters is given by $G=(1-P) c_{s} 0 /\left(P \cdot c_{1,0}\right)$. Parallel to the definition of $I_{y}$, the dimensionless overall electrode current $I_{z}$ is defined as the total electrode current relative to the stoichiometric capacity of the electrode and the time constant of the electrode particles

$$
I_{z}=\int_{0}^{1} I_{y}(Z) d Z=\frac{i_{z}}{\mathbf{F} \cdot c_{s}{ }^{\circ}(1-P) \cdot l} \cdot \frac{r^{2}}{D_{s}{ }^{\circ}}
$$

[ $I_{\mathrm{z}}$ is identical to $1 / Q$ as defined in Ref. (10).]

Length is measured in different units for directions parallel and perpendicular to the pore surface in order to obtain length parameters that always vary between 0 and 1 , regardless of the physical dimensions of the pore system. Also concentration is measured relative to different standards in the electrode and the electrolyte phases.

The porous insertion electrode is thus described by the local variables $C(Z), E(Z), I_{y}(Z)$ and $X(Y, Z)$, and transport in this system is governed by Eq. [12], [17], and [18] together with an expression for the dependence of $E$ on $C$ and $X^{*}$

$$
E=E^{0}+\ln \left(\left(1-X^{*}\right) / X^{*}\right)+\ln (C)-f\left(X^{*}-0.5\right)
$$

The transport equations are subject to the following boundary equations:

$$
\begin{gathered}
\left.\nabla_{\mathrm{Y}} X\right|_{\mathrm{Y}=0}=0 \\
\left.\nabla_{\mathrm{Y}} X\right|_{\mathrm{Y}=1}=-I_{\mathrm{Y}} / U_{\mathrm{S}} \cdot n_{\mathrm{g}} \\
\left.\frac{\partial C}{\partial Z}\right|_{Z=0}=\left.\frac{\partial E}{\partial Z}\right|_{Z=0}=0 \\
C(Z=1)=1 \\
E(Z=1)=E^{*}
\end{gathered}
$$

where $E^{*}$ is the (dimensionless) potential difference between the electrode phase and the electrolyte just outside the pore. 


\section{Computational Methods}

This set of coupled, nonlinear differential equations cannot be solved analytically in the general case. Numerical approximations to the solution can, however, be obtained by finite difference methods $(16,17)$, where the continuous variables are replaced by a set of approximate values defined at certain mesh points, and where the differential operators are replaced with difference operators.

A linearization of $X^{*}(Z)$ on $T$ and $I_{\mathrm{y}}(Z)$ is obtained at each time step from the numerical solution of Eq. [12] together with the boundary conditions specified above. The resulting set of coupled, nonlinear equations in $C, X^{*}, E$, and $I_{y}$ is solved simultaneously with no further decoupling or linearizations, following the iterative scheme proposed by Brumleve and Buck (18). Using this technique, very stable numerical solutions are obtained, which are only slightly affected (less than $2 \%$ ) by changing the number of discretization points between 10 and 100 in any dimension.

The boundary condition [25] used above, represents the potentiostatic case, where the charge or discharge of the electrode proceeds at controlled potential. In practice, however, batteries are operated at conditions more similar to the galvanostatic case, where the electrodes are charged or discharged with a controlled current. To account for this case, an iterative loop is added to the calculations at each time step, in which the potential just outside the pore $\left(E^{*}\right)$ is adjusted until the specified value of the electrode current $\left(I_{z}\right)$ is obtained.

\section{Results}

The salient features of the model electrode system under load are illustrated with results from constant current simulations. The material properties used in these simulations are those of a porous titanium disulfide electrode filled with an ideal organic electrolyte.

Lithium transport in TiS $_{2}$ crystals is anisotropic as there is virtually no mobility for lithium ions in the direction of the crystallographic c-axis (23). Consequently, transport in the typically disk shaped $\mathrm{TiS}_{2}$ particles is adequately described by the cylinder model, as they fulfill the requirements of no significant transport across the ends of the cylinders, even though the "cylinders" are very short.

A reasonable approximation to the emf of $\mathrm{Li}_{x} \mathrm{TiS}_{2}$ vs. a lithium reference electrode $\left(c_{1}=c_{1}{ }^{\circ}\right)$ as measured at this laboratory is given by

$$
\begin{aligned}
\pi_{L_{x} T_{i S_{2}}}-\pi_{L i\left(\mathrm{cl}=c_{1}\right)} & =2.17 \mathrm{~V}+\frac{R T}{F}\left[\ln \left(\frac{1-X^{*}}{X^{*}}\right)\right. \\
& \left.+\ln (C)-16.2\left(X^{*}-0.5\right)\right]
\end{aligned}
$$

This expression does not reproduce the fine structure of the emf curve [see (19) and (20)], but the overall approximation is satisfactory for practical purposes.

The values of the transport parameters used in the simulations are listed in Table I. $D_{ \pm}$and $t_{+}$are experimental values for a $1 \mathrm{M}$ solution of $\mathrm{LiClO}_{4}$ in propylene carbonate. All transport parameters are taken to be independent of the composition of the phases although this condition is hardly ever met by practical electrode systems. Detailed experimental determinations of the variation of these parameters with concentration are, however, not available at present, and as this variation is only considered to introduce second-order effects, the idealized case is chosen to illustrate the behavior of the model.

The magnitude of the geometrical parameters also listed in Table I are characteristic values of a battery optimized for traction purposes (9).

On Fig. 3 the equilibrium emf curve is compared with a simulated constant current discharge. The con-

Toble I. Simulation parameters

Transport data, electrolyte:

Transport number: $t_{+}=0.20$

Salt diffusion coefficient

$D \pm=2.58 \times 10^{-8} \mathrm{~cm}^{2} / \mathrm{sec}$

Ref. (21)

$D_{+}=1.61 \times 10^{-8} \mathrm{~cm}^{2} / \mathrm{sec}$

Ref. (22)

$D_{-}=6.45 \times 10^{-8} \mathrm{~cm}^{2} / \mathrm{sec}$

Transport data, electrode:

Chemical diffusion coefficient of $\mathrm{Li}$ in $\mathrm{Li}_{x} \mathrm{TiS} 2$

$$
D_{0^{\circ}}=D_{0}=10^{-10} \mathrm{~cm}^{2} / \mathrm{sec} \quad \text { Ref. (23) }
$$

Geometrical data: *

Electrode thickness

Particle diameter

Porosity

Discharge current density

Electrolyte concentration (start)

Saturation concentration of electrode

$$
\begin{aligned}
& =0.1 \mathrm{~cm}(l=0.05 \mathrm{~cm}) \\
& =1 \cdot 10^{-4} \mathrm{~cm}\left(r=5 \cdot 10^{-5} \mathrm{~cm}\right) \\
& =0.35 \\
& =i_{2}=5 \mathrm{~mA} / \mathrm{cm}^{2} \\
& =c_{1,0}=1 \times 10^{-3} \mathrm{~mol} / \mathrm{cm}^{3} \\
& =c_{8^{0}}=2.5 \times 10^{-2} \mathrm{~mol} / \mathrm{cm}^{3}
\end{aligned}
$$

- The geometrical data correspond roughly to those of Ref. (9).

stant current load chosen, $5 \mathrm{~mA} / \mathrm{cm}^{2}$, is equivalent to a stoichiometric discharge time of $4: 21 \mathrm{hr}$, which is reasonable for traction purposes. The maximal coulombic efficiency predicted for this load is $80 \%$. The reason for this limitation is the evolution of an electrolyte depletion region during discharge, as shown in Fig. 4 , where some of the dimensionless parameters of the model are depicted as functions of the length coordinate $(Z)$ at the three different degrees of discharge indicated on Fig. 3. Figure 4a shows how the electrolyte concentration in the center of the electrode approaches zero during discharge, as the transport of lithium ions from the bulk electrolyte cannot keep pace with the lithium ion consumption. This is a consequence of the relatively high anion mobility. Simultaneous with this electrolyte depletion, the surface of the electrode particles in the outer parts of the electrode gradually becomes saturated with lithium (Fig. $4 \mathrm{~b})$. When the surface concentration cannot be increased further, the discharge current in this region is limited to a value just large enough to support the equilibration process until all the underlaying electrode material eventually becomes saturated. The electrode current is thus limited both in the depletion region, due to the low lithium ion concentration, and in the saturation region. As these regions approach each other during the discharge, the major part of the electrode reaction is confined to a narrowing zone (Fig. $4 c$ ), until it finally becomes impossible to discharge the electrode further with the chosen current.

The charge transfer resistance of a titanium disulfide electrode in $\mathrm{LiClO}_{4} / \mathrm{PC}$ electrolyte is less than $150 \Omega \mathrm{cm}^{2}$ for vaules of $X$ between 0 and 1 (23). From the current distributions of Fig. $4 \mathrm{c}$, it can be seen that

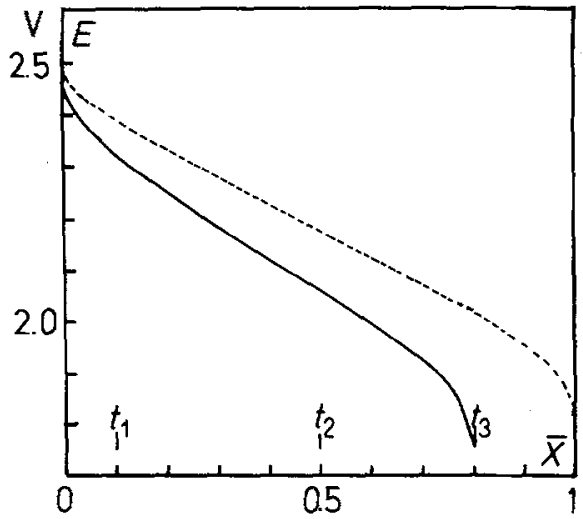

Fig. 3. Equilibrium emf curve (broken line) as given by Eq. [26] compared with a simulated constant current discharge curve. Simulation parameters are given in Table $\mathrm{I}$. 

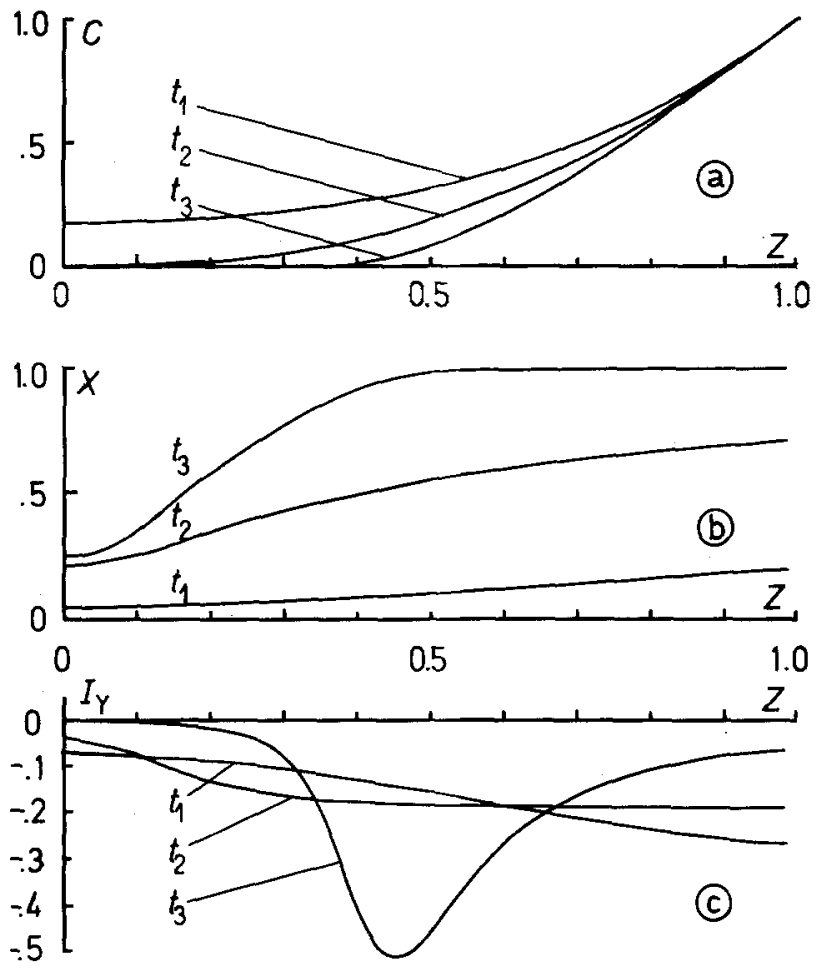

Fig. 4. Spatial variation of: (a) electrolyte concentration (C), (b) surface concentration at electrode/electrolyte interface $\left(X^{*}\right)$, and $(c)$ current density at electrode/electrolyte interface $\left(I_{Y}\right)$. The curves correspond to the different degrees of discharge indicated on Fig. $3\left(t_{1} \simeq 10 \%, t_{2} \simeq 50 \%, t_{3} \simeq 79 \%\right)$.

inclusion of charge transfer resistance in the model would change the electrode polarization less than 20 $\mathrm{mV}$, which is in agreement with the initial assumption of negligible influence of the charge transfer overvoltage on the electrode performance.

For low current loads or thin electrodes this depletion phenomenon will not occur, but still the electrode utilization can be limited due to the loading of the electrode particles as described in Ref. (10). This is illustrated in Fig. 5, where the electrode utilization is shown as function of the electrode thickness $(l)$ and the constant current load $\left(i_{z}\right)$. The loci of $90 \%, 50 \%$, and $10 \%$ maximal coulombic utilization is shown both as they emerge from the porous electrode simulations and as predicted from plane insertion electrode theory (10). For a fixed electrode current density it can be

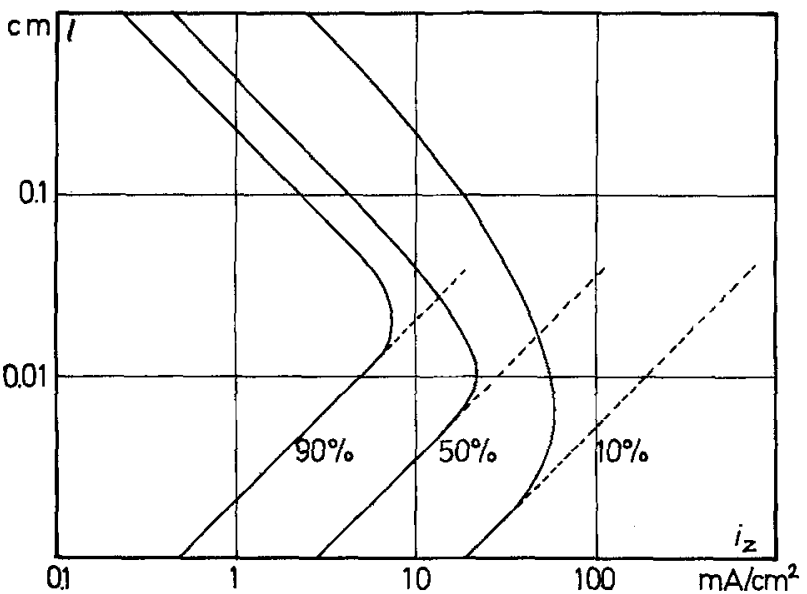

Fig. 5. Maximal electrode utilization as function of electrode thickness (I), and electrode current $\left(i_{z}\right)$. All other parameters are as given in Table 1 . The dashed lines are utilizations predicted from plane electrode theory (10).

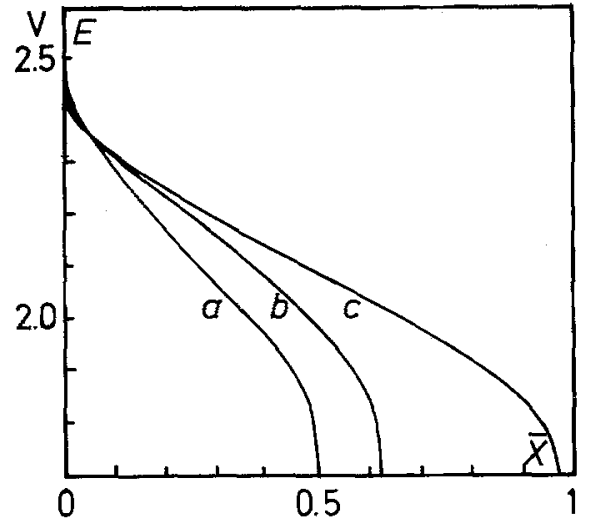

Fig. 6. Simulated discharge curves for varying anion diffusion coefficients $\left[D_{-}=6.45 \times 10^{-6} \mathrm{~cm}^{2} / \mathrm{sec}(\mathrm{a}) ; 1.79 \times 10^{-7} \mathrm{~cm}^{2}\right.$ ) $\sec \left(\right.$ b) $; 1.63 \times 10^{-8} \mathrm{~cm}^{2} / \mathrm{sec}$ (c) $]$. Electrode thickness $I=0.1$ $\mathrm{cm}$, electrode current $i_{\mathrm{z}}=4.2 \mathrm{~mA} / \mathrm{cm}^{2}$, all other parameters as

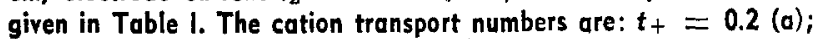
0.9 (b), 0.99 (c).

seen that increasing the electrode thickness first increases the electrode utilization as the average loading of the individual electrode particles decreases. Later, when electrolyte depletion limits the electrode utiliza. tion, further increase of the electrode thickness only decreases the average utilization, as the added material is not utilized.

As mentioned above, changes in the cation transport number $\left(t_{+}\right)$are expected to influence the maximal electrode utilization. This is illustrated in Fig. 6, where three simulated discharge curves are compared. The only parameter varied between the simulations is the anion mobility, whereas the cation mobility, geometrical parameters, and current load are kept constant. It can be seen that even though the overall electrolytic conductivity is decreased, a substantial increase in electrode utilization can be obtained when the cation transport number is raised toward unity. From these examples it is concluded that the porous insertion electrode with conventional organic electrolyte is mainly limited by diffusion either in the pore or in the electrode particles. Optimization of these electrodes therefore requires careful consideration of the coupled transport in both the electrode and the electrolyte phases. Furthermore it can be concluded that better utilization can be obtained using electrolytes with unity transport number of the active cation (e.g., solid electrolytes), if a satisfactory solution to the contact problems between a solid electrode phase and a nonliquid electrolyte can be found.

\section{Acknowledgments}

The authors wish to acknowledge the inspiration gained from discussions with colleagues participating in the joint British-Danish Energy Research Programme (Contracts 315, 316-78-1 EE DK, UK) and the financial support to one of us (Keld West) from the Danish Department of Energy.

Manuscript submitted Dec. 29, 1980; revised manuscript received ca. Dec. 1, 1981.

Any discussion of this paper will appear in a Discussion Section to be published in the June 1983 JourNaL. All discussions for the Iune 1983 Discussion Section should be submitted by Feb. 1, 1983.

\section{IIST OF SYMBOLS}

$c_{1}$ anion and cation concentration in electrolyte $c_{1,0}$ anion and cation concentration in electrolyte at time $T=0$ or at $z=l$

$c_{s}$ concentration of inserted species in electrode

$c_{s, 0}$ concentration of inserted species in electrode at $T=0$ 
$c_{s}{ }^{\circ}$ saturation concentration of inserted species in electrode

$c_{s}{ }^{*}$ value of $c_{s}$ at electrode/electrolyte interface

$C$ dimensionless concentration in electrolyte = $c_{1} / c_{1,0}$

$D_{\mathrm{s}}$ diffusion coefficient of inserted species in electrode phase

$D_{\mathrm{s}}{ }^{o}$ reference value of $D_{\mathrm{s}}$

$D_{+}$diffusion coefficient of cation in electrolyte

$D_{-}$diffusion coefficient of anion in electrolyte

$E$ dimensionless potential difference between electrode and electrolyte $=(\pi-\phi) \cdot \mathbf{F} / R T$

$E^{\circ}$ constant in Eq. [20]

$E^{*}$ dimensionless potential difference between electrode phase and electrolyte just outside the pore

$f$ "Interaction" parameter in Eq. [10]

F Faraday's number

$g$ ratio between circumference and cross-sectional area of pore

$G$ dimensionless geometric factor $=$ $g \cdot r \cdot c_{\mathrm{s}} / c_{1,0} \cdot n_{\mathrm{g}}$

$i_{y} \quad$ current density at particle/electrolyte interface

$I_{y}$ dimensionless current density at electrode/electrolyte interface $=i_{\mathrm{y}} \cdot r \cdot n_{\mathrm{g}} / \mathbf{F} \cdot c_{\mathrm{s}}{ }^{\circ} \cdot D_{\mathrm{s}}{ }^{\circ}$

overall electrode current density

$I_{\mathrm{z}}$ dimensionless current density at electrode surface $=i_{\mathrm{z}} \cdot r^{2} / \mathbf{F} \cdot c_{\mathrm{s}}{ }^{0} \cdot D_{\mathrm{s}}{ }^{0}(1-P) \cdot l$

distance from center to surface of electrode

$n_{\mathrm{g}} \quad$ geometry type $(1,2,3)$

$P$ porosity

$r$ distance from center to surface of electrode particle

$R T$ product of gas constant and absolute temperature

$t$ time

$t_{+}$cation transport number

$T$ dimensionless time $=t \cdot D_{s} 0 / r^{2}$

$U_{+}$dimensionless mobility $=\left(D_{+} / D_{\mathrm{s}} \mathrm{o}\right)(l / r)^{2}$

$U_{-}$dimensionless mobi 1 ity $=\left(D_{-} / D_{s}^{0}\right)(l / r)^{2}$

$U_{\mathrm{s}}$ dimensionless mobility $=D_{\mathrm{s}} / D_{\mathrm{s}}{ }^{\circ}$

$X$ dimensionless concentration in electrode phase $=$ $c_{s} / c_{s}{ }^{\circ}$

$X^{*} \quad$ value of $X$ at electrode/electrolyte interface

$\bar{X}$ mean value of $X$

$y$ spatial variable across electrode particle (perpendicular to electrode/electrolyte interface)

$Y$ dimensionless spatial variable $=y / r$

$z$ spatial variable across electrode (parallel to the pore)

$Z \quad$ dimensionless spatial variable $=z / l$

$\phi$ potential in electrolyte $\phi^{*} \quad$ potential in electrolyte just outside the pore

$\pi \quad$ potential in electrode phase (Fermi potential)

$(\pi-\phi)^{\theta}$ constant in Eq. [10]

\section{REFERENCES}

1. M. S. Whittingham, Prog. Solid State Chem., 12, 41 (1978).

2. D. W. Murphy and F. A. Trumbore, J. Cryst. Growth, 39, 185 (1977)

3. M. S. Whittingham, This Journal, 123, 315 (1976).

4. M. S. Whittingham, Mater. Res. Bull., 13, 959 (1978)

5. J. S. Gore and C. R. Walk, U.S. Pat. 3,929,504 (1975).

6. D. W. Murphy, P. A. Christian, F. J. DiSalvo, and J. V. Waszczak, Inorg. Chem., 18, 2800 (1979).

7. G. Pistoia, M. Pasquali, and P. Fiordiponti, Ext. Abstract, 32nd I.S.E. Meeting (1981).

8. A. J. Jacobson, R. R. Chianelli, and M. S. Whittingham, This Journal, 126, 2272 (1979).

9. L. H. Gaines, R. W. Francis, G. H. Newman, and B. M. L. Rao, 11th Intersociety Energy Conversion Engineering Conference, Stateline, NV (1976).

10. S. Atlung, $K$. West, and T. Jacobsen, This Journal, 126, 1311 (1979).

11. R. deLevie, Adv. Electrochem. Electrochem. Eng., 6, 329 (1967).

12. J. Newman and W. Tiedemann, AIChE J., 21, 25 (1975).

13. M. Armand, Thesis, University of Grenoble (1978).

14. R. Pollard and J. Newman, Electrochim. Acta, 25, 315 (1980).

15. J. Crank, "Mathematics of Diffusion," p. 186, Oxford University Press, (1956).

16. S. W. Feldberg, Electroanal. Chem., 3, 199 (1969).

17. A. R. Mitchel," "Computational Methods in Partial Differential Equations," John Wiley \& Sons, Inc. (1969)

18. T. R. Brumleve and R. P. Buck, J. Electroanal. Chem. Interfacial Electrochem., 49, 1 (1978).

19. A. H. Thompson, This Journal, 126, 608 (1979).

20. T. Jacobsen, K. West, and S. Atlung, ibid., 126, 2169 (1979).

21. R. Jasinski, Adv. Electrochem. Electrochem. Eng., 8, 291 (1972).

22. J. M. Sullivan, D. C. Hanson, and R. Keller, This Journal, 11\%, 779 (1970).

23. K. West, T. Jacobsen, B. Zachau-Christiansen, and S. Atlung, Ext. Abstract, A 11, 32nd I.S.E. Meeting (1981).

\title{
Use of Liquid Chromatography for Studying Interfacial Properties of Inorganic Solutes Relevant to Reverse Osmosis Separations
}

\author{
Yutaka Taketani, Takeshi Matsuura, and S. Sourirajan \\ National Research Council of Canada, Division of Chemistry, Ottawa, Ontario, Canada K1A OR9
}

\section{ABSTRACT}

High performance liquid chromatography (HPLC) offers a means of investigating the properties of inorganic solutes at polymer (cellulose acetate)-aqueous solution interfaces. Equilibrium constants of solutes distributed between interfacial- and bulk-phases and restricted diffusion of solutes in the interfacial region can be evaluated using retention volume and half-width data from HPLC experiments. These data offer a means of correlating and predicting the differences in free energy parameters governing the reverse osmosis separation of inorganic solutes.

The study of the properties of water at the polymersolution interfacial region (interfacial water) is fundamental for understanding the transport of solute and solvent water in a reverse osmosis membrane. This is particularly so since the properties of water in the membrane pore are practically the same as those in the interfacial water, as long as the membrane pore size is sufficiently small. It is considered that high

Key words: reverse osmosis, cellulose acetate, inorganic solutes, interfacial properties, HPLC. performance liquid chromatography (HPLC) in which solvent water flows through a column packed with a polymer material simulates the interfacial phenomena taking place during the reverse osmosis transport involving membranes made of that polymer material. By applying the already well-established theories of chromatography to the analysis of the experimental retention time and related data, quantities characterizing interfacial properties, such as the equilibrium constant for the solute distributed between the bulk and 